Part of Journal of Research of the National Bureau of Standards, Volume 25, November 1940

\title{
RECOMBINATION AND ELECTRON ATTACHMENT IN THE $F$ LAYERS OF THE IONOSPHERE
}

\author{
By Fred L. Mohler
}

\section{ABSTRACT}

Observational data for the ionosphere at Washington have been used as a basis for computations. The rate of recombination at night is roughly proportional to the square of the electron concentration, but the apparent recombination coefficient, $\alpha^{\prime}$, tends to increase with decreasing electron concentration over the half period of a sunspot cycle. 'This fact and the observed variation of $\alpha^{\prime}$ with height indicate that electrons disappear both by recombination and by negativeion formation. The daily variation of electron concentration indicates that the $F_{2}$ level is far above the level where the rate of production of electrons is a maximum in midsummer and that the $F_{1}$ layer is near the level where the rate is a maximum. It is assumed that both layers come from the ionization of atomic oxygen.

If $\alpha^{\prime}$ is a function of the pressure, there will be a maximum electron concentration at a pressure, $p$, given by the relation

$$
\frac{p}{p_{0}}=\frac{\alpha}{\alpha^{\prime}} f(\lambda)
$$

where $p_{0}$ is the pressure at the $F_{1}$ level, $\alpha$ is the electronic-recombination coefficient, and $\lambda$ is the ratio of negative ions to electrons. $f(\lambda)$ is 1 , if $\lambda$ is small, or a linear function of $p$. In midsummer, $p$ is $4 \times 10^{-8} \mathrm{~mm}$ of mercury. $p / p_{0}=0.027$ and $\alpha=2 \times 10^{-12}$, in good agreement with the theory of recombination. A seasonal variation of $p / p_{0}$ can be explained, if $f(\lambda)$ is not always unity, as $\lambda$ is very sensitive to temperature. The seasonal variation in electron concentration can be accounted for by the change in pressure. The pressure variation also accounts for the fact that $F_{2}$ changes much more than $F_{1}$ over a sunspot cycle. The effective collision area for electron attachment to oxygen atoms is $6.5 \times 10^{-21} \mathrm{~cm}^{2}$.

\section{CONTENTS}

I. Introduction Page

II. Observational data

III. Elementary theory

IV. Recombination and electron attachment_...

V. Daytime variation of electron concentration

VI. Pressure at the $F_{2}$ level

VII. Numerical results

VIII. Conclusion.

\section{INTRODUCTION}

Phenomena of the ionosphere have been the subject of many theoretical investigations. The conventional theory is that electrons are produced by photoionization by solar radiation and disappear by recombination at a rate proportional to the square of the electron concentration. The different layers of ionization are usually ascribed to different absorption processes. This theory gives a very satisfactory explanation of the daily and annual variations of the $\dot{E}$ and $F_{1}$ 
layers, ${ }^{1,2}$ but the $F_{2}$ layer varies in an apparently erratic manner that has never been adequately explained. This layer is characterized by a very slow rate of recombination, and Bradbury ${ }^{3}$ has suggested that the $F_{2}$ maximum in electron concentration may come from the same ionization process that gives $F_{1}$ purely as a consequence of the slow recombination. Recombination in the ionosphere probably involves both spontaneous recombination of electrons and positive ions and negative-ion formation followed by ionic recombination, and the relative importance of the latter process will increase with increasing pressure. This paper reports an attempt to deduce the nature of the recombination processes from observational data on the $F$ region.

\section{OBSERVATIONAL DATA}

Ionosphere characteristics are commonly published as plots of the critical radio penetration frequency and minimum virtual height

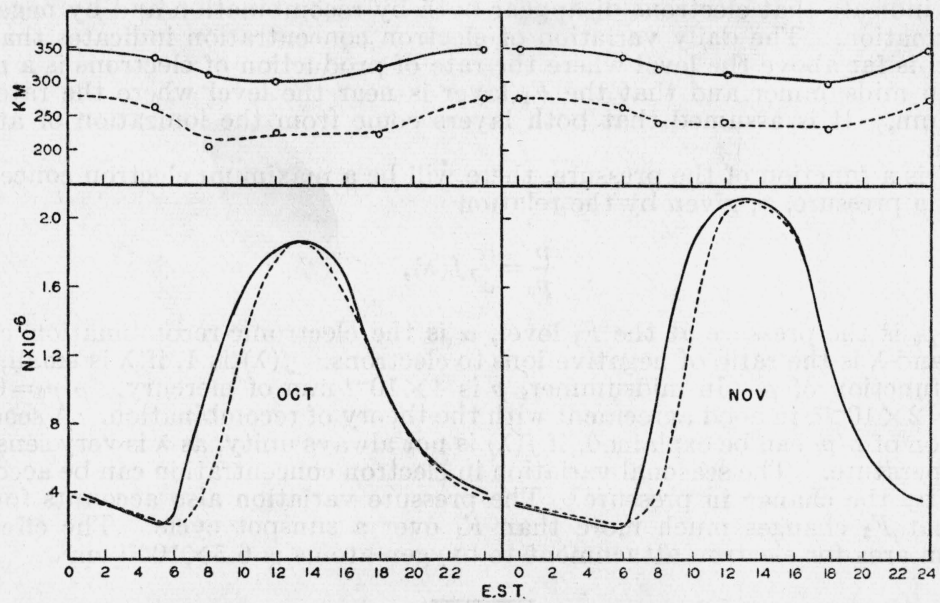

FigURE 1.-Mean values of electron concentration and true height of the $F_{2}$ layer for Washington, October and November 1938.

A bove, the full line is true height of the maximum, and the broken line is the height where $N_{0}$ is half the maximum value. Below, the broken line is the theoretical curve for the conventional theory with $\alpha^{\prime}=$ $0.8 \times 10^{-10}$ in October and $1.0 \times 10^{-10}$ in November.

versus the time of day. Monthly mean values for several stations are published periodically. ${ }^{4}$ The critical frequency depends on the number of free electrons per cubic centimeter, $N_{e}$, which is here computed by the equation

$$
N_{e}=0.0124 f^{2} \text {, }
$$

where $f$ is the critical frequency in kilocycles for the ordinary wave. The question as to the proper coefficient in eq 1 is not important for this work.

The true height is not simply related to the virtual height, but methods ${ }^{5}$ are now available for the computation of true height, and

\footnotetext{
1 E. V. Appleton, Proc. Roy. Soc. (London) [A] 162, 451 (1937).

2 E. O. Hulbert, Phys. Rev. 55, 639 (1939)

3 Ter. Mag. and Atmos. Elec. 43, 55 (1938).

- National Bureau of Standards data for Washington, D. C., by Gilliland, Smith, and Kirby, appear monthly in the Proceedings of the Institute of Radio Engineers.

- F. H. Murray and J. B. Hoag, Phys. Rev. 51, 333 (1937).
} 
$\mathrm{N}$. Smith has made rather extensive computations of true heights as a function of frequency for the ionosphere at Washington during the past few years, and I am indebted to him for the opportunity to use some of this unpublished material.

A few months in 1938 have been selected as a basis for computations. Figures 1 and 2 give the number of electrons and true height as a function of the time of day for different months. This is for a period of maximum solar activity. In years of minimum solar activity the number of electrons in the $F_{2}$ layer was about one-fourth of the 1938 value, the daily curve broader and flatter.

Figure 3 is a plot of the vertical distribution of electrons above the $E$ layer, as computed by Smith. The $F_{1}$ layer does not appear in October 1938 but is found in years of low solar activity.

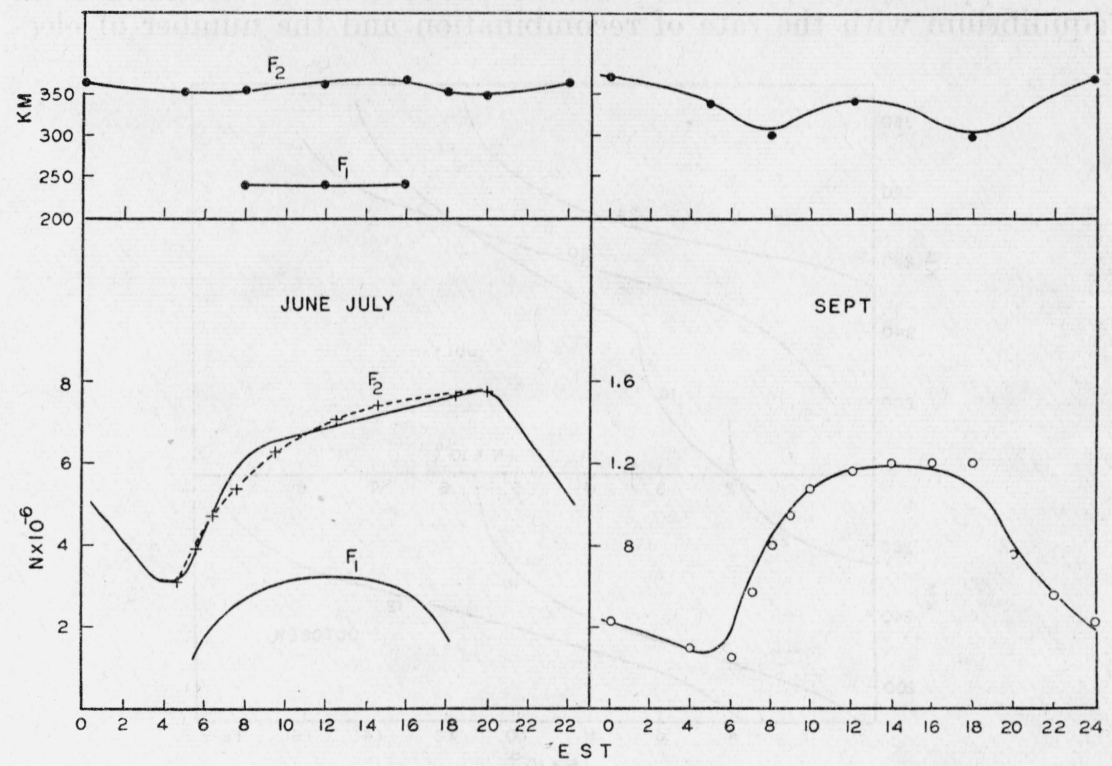

FigURE 2.-Mean values of electron concentration and true height for Washington, June, July, and September 1938.

Above, true height of the maxima ( $F_{1}$ is not well defined in September); below, full lines give observed values of $N_{0}$ (two scales). The broken line and crosses are computed on the assumption that absorption is negligible and electrons disappear purely by electron attachment; circles, on the assumption of pure recombination with no absorption.

\section{ELEMENTARY THEORY}

If $a$ is the atomic-absorption coefficient of the ionizing radiation, then the flux,.$J$, of ionizing quanta per square centimeter per second is

$$
J=J_{0} e^{-N a},
$$

where $J_{0}$ is the flux outside the atmosphere and $N$ is the number of atoms in a square-centimeter column extending from the point where $J$ is evaluated to the outside of the atmosphere.

The radiation is reduced to $1 / e$ in a column of gas containing $1 / a$ atoms per square centimeter and weighing $M / a$ grams, where $M$ is the weight of one atom. For vertical sunlight, $M / a$ is the partial 
pressure in grams per square centimeter, and $M / 1.35 a$ is the partial pressure in millimeters of mercury at the level where the radiation is reduced to $1 / e$. The number of atoms per cubic centimeter at this pressure is $0.725 \times 10^{19} \mathrm{M} / a T$, and the rate of production of electrons per cubic centimeter per second is

$$
q=\frac{0.725 \times 10^{19} M J_{0}}{T} e^{-1},
$$

and for any partial pressure, $p$,

$$
q=\frac{0.725 \times 10^{19} M J_{0}}{T} \frac{p}{p_{1}} e^{-\frac{p}{p_{1}}}
$$

where $p_{1}=M / 1.35 a$. If the rate of production of electrons is in equilibrium with the rate of recombination and the number of elec-

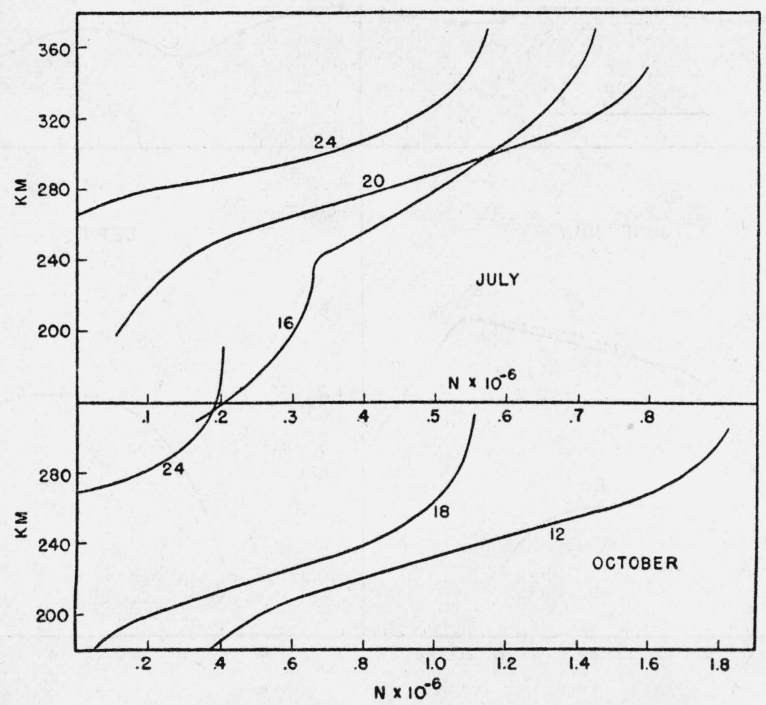

FIGURE 3.-Electron concentration as a function of the height for Washington, July and October 1938 at the hours indicated on the curves.

From calculations by N. Smith.

trons per cubic centimeter, $N_{e}$, is assumed equal to the number of positive ions,

$$
\left.\begin{array}{c}
q=\alpha N_{\bullet}^{2} \\
N_{\bullet}=\frac{K^{1 / 2}}{\alpha^{1 / 2}} \frac{p^{1 / 2}}{p_{1}^{1 / 2}} e^{-\frac{\circ}{2 p_{1}}} \\
K=0.725 \times 10^{19} M J_{0} / T
\end{array}\right\} .
$$

For the case of oblique sunlight entering the atmosphere at a zenith angle, $Z$, the light is reduced to $1 / e$ at a pressure $p_{1} \cos Z$, and the maximum rate of production of ions is reduced by the factor $\cos Z$. 
In an atmosphere of atoms of mass, $M$, the relation between pressure, $p$, and altitude, $h$, can be expressed

$$
\left.\begin{array}{l}
\frac{p_{1}}{p_{2}}=e^{\left(h_{2}-h_{1}\right) / H} \\
H=k T \times 10^{-5} M g
\end{array}\right\},
$$

where $k$ is Boltzman's constant, $g$ is the gravitational constant, and $H$ is the height, in kilometers, in which the pressure is reduced to $1 / e$ (commonly called the scale height).

It will be assumed in numerical computations that atomic oxygen gives rise to $F$-layer ionization. Wulf and Deming ${ }^{6}$ have shown that the dissociation of oxygen must be nearly complete above $100 \mathrm{~km}$ and estimate the number of oxygen atoms per cubic centimeter at $100 \mathrm{~km}$ as $10^{12}$. The dissociation of oxygen produces a sharply defined transition both in chemical composition and temperature near the $100-\mathrm{km}$ level. If it is assumed that gases are in separate gravitational equilibrium above $100 \mathrm{~km}$, then for atomic oxygen

$$
H=T / 18.8 \text {. }
$$

The theoretical value of the atomic absorption coefficient of oxygen ${ }^{7}$ at the photoelectric threshold, $910 \mathrm{~A}$, is $1.25 \times 10^{-17}$, and $p_{1}$ for vertical sunlight is $1.56 \times 10^{-6} \mathrm{~mm}$.

It is generally considered that at the $F_{1}$ level there will be a large proportion of atomic oxygen negative ions, and ionic recombination may be important. The rate of absorption, $q$, gives the rate of production of positive ions, and if $\lambda$ is the ratio of negative ions to electrons,

$$
N_{+}=N_{e}+N_{-}=(1+\lambda) N_{e},
$$

and for equilibrium between production of ions and recombination

$$
\begin{gathered}
q=\alpha N_{e} N_{+}+\alpha_{i} N_{-} N_{+} \\
q=\left[(1+\lambda) \alpha+\lambda(1+\lambda) \alpha_{i}\right] N_{e}^{2}=\alpha^{\prime} N_{e}^{2},
\end{gathered}
$$

where $\alpha_{i}$ is the ionic recombination coefficient and $\alpha^{\prime}$ the apparent coefficient for recombination. Appleton and Sayers ${ }^{8}$ emphasize that since the daily and annual variation of the $F_{1}$ layer conforms to the simple recombination theory, $\alpha^{\prime}$ must be a constant and $\lambda$ must be independent of pressure at the $F_{1}$ level.

The rate of recombination in the $F_{2}$ layer is very much less than in the $F_{1}$ layer, so it is necessary to assume that $\lambda$ decreases with the pressure above $F_{1}$. It commonly has been assumed that spontaneous recombination of electrons and positive ions will be predominant in the $F_{2}^{\prime}$ layer, because the pressure is very low. However, in contrast to the $F_{1}$ layer, the daily and annual variations of $F_{2}$ ionization give no evidence that $\alpha^{\prime}$ is independent of pressure at this height.

- Ter. Mag. and Atmos. Elec. 43, 283 (1938).

7 D. R. Bates, R. A. Buckingham, H. S. W. Massey, and J. J. Unwin, Proc. Roy. Soc. (London) [A] 170, 322 (1939).

8 Union, Rad. Sci. Int. Proc., p. 272 (1938). 


\section{IV." RECOMBINATION AND ELECTRON ATTACHMENT} then

If electrons disappear by simple recombination during the night,

$$
\frac{d N_{e}}{d t}=-\alpha^{\prime} N_{e}^{2}
$$

and the integral of this

$$
\frac{1}{N_{2}}-\frac{1}{N_{1}}=\alpha^{\prime}\left(t_{2}-t_{1}\right)
$$

where $N_{1}$ and $N_{2}$ are the number of electrons at times $t_{1}$ and $t_{2}$. Figure 4 gives plots of $1 / N_{e}$ versus $t$ for the data of figures 1 and 2 . The

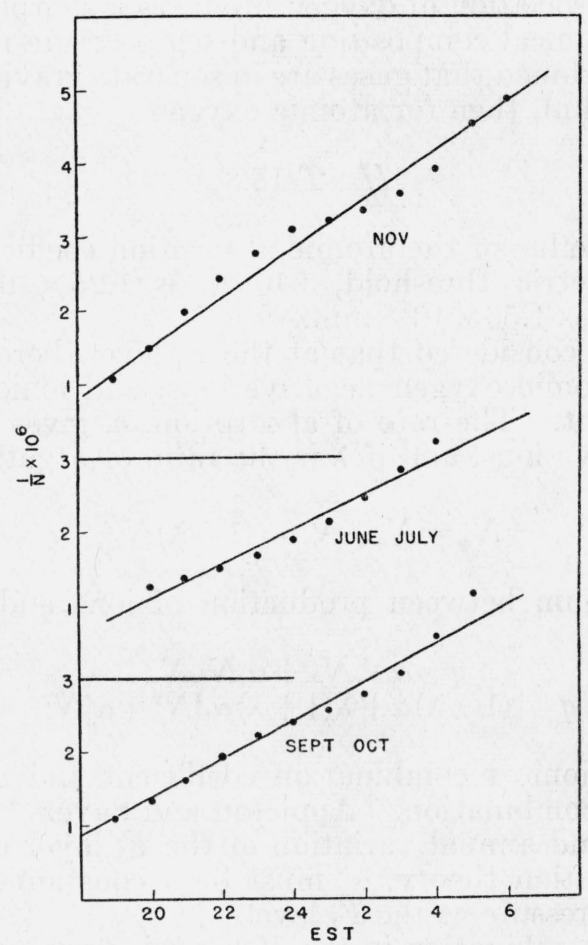

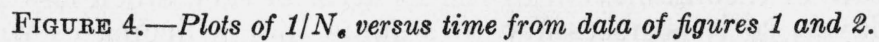

5. Slopes of the lines [give $\alpha^{\prime}=0.95 \times 10^{-10}, 10.66 \times 10^{-10}$, and $\left\{0.72 \times 10^{-10}\right.$, from top to bottom.

slopes of the straight lines give values of $\alpha^{\prime}$, ranging from $0.66 \times 10^{-10}$ to $0.95 \times 10^{-10}$.

Appleton ${ }^{9}$ has pointed out that the rate of recombination during the daytime can be evaluated from pairs of values of $N_{e}$ and of $d N_{\epsilon} / d t$ at equal intervals before and after noon by the equation,

$$
\left(\frac{d N_{e}}{d t}\right)_{a . m .}-\left(\frac{d N_{e}}{d t}\right)_{p . m .}=\left[\left(N_{e}^{2}\right)_{p . m .}-\left(N_{e}^{2}\right)_{a . m .}\right] \alpha^{\prime},
$$

- Proc. Roy. Soc. (London) [A] 162, 451 (1937). 
where subscripts a. m. and p. m. denote values at equal time intervals before and after noon. The October curve (fig. 1) gives a mean value of $\alpha^{\prime}=0.9 \times 10^{-10} \pm 0.2 \times 10^{-10}$, as compared with the nighttime value of $0.7 \times 10^{-10}$. Values based on sunrise and sunset values of the slopes and ordinates for 20 months in the period $1937-39$ give $\alpha^{\prime}=0.9 \times$ $10^{-10} \pm 0.3 \times 10^{-10}$ and in the same period the mean nighttime value is about $0.7 \times 10^{-10}$. However, nighttime values for years of low solar activity (1934 and 1935) are very much larger than this, and the values of $\alpha^{\prime}$ seem to be definitely correlated with the amount of ionization. Figure 5 is a plot of $\alpha^{\prime}$ versus midnight values of $1 / N_{e}$ for 30 months in the period 1934-39. Although the points are widely scattered, the plot indicates that $\alpha^{\prime}$ is inversely proportional to $N_{e}$. This would suggest that electrons disappear by attachment rather

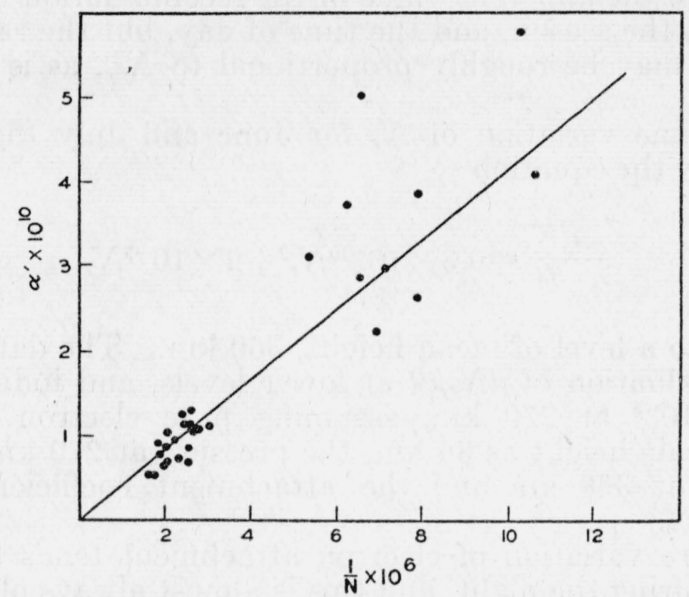

Frgure 5.-Values of the apparent recombination coefficient as a function of $1 / N_{e}$ at midnight for years 1934 to 1939.

than recombination. The time variation of electron concentration during the night would be

$$
\frac{d N_{e}}{d t}=-\beta N_{o} N_{e}=-B p N_{e}
$$

where $N_{o}$ is the number of oxygen atoms per cubic centimeter; $p$, the partial pressure of oxygen atoms; $\beta$ and $B$, constants. The integral of this is

$$
\ln N_{1}-\ln N_{2}=B p\left(t_{2}-t_{1}\right) \text {. }
$$

Values for midnight and $4 \mathrm{a}$. $\mathrm{m}$. for the 30 months considered above give values of $B p$ which are far from constant but show no systematic variation with $N_{e}$. The mean value is $B p=2.9 \pm 0.8 \times 10^{-5}$.

It seems impossible to admit, however, that electrons disappear purely by electron attachment, for this would not give a well-defined maximum in the vertical distribution of electrons, such as is observed, but rather a gradual exponential variation proportional to $e^{-p}$. It is of interest to investigate the possibility that electrons disappear 
both by recombination and attachment. Then the nighttime variation would be

and, by eq 8 ,

$$
-\frac{d N_{e}}{d t}=\alpha N_{e} N_{+}+B p N_{e},
$$

$$
-\frac{d N_{e}}{d t}=\left[\alpha(1+\lambda)+\frac{B p}{N_{e}}\right] N_{e}^{2},
$$

where $\lambda$ is as before the ratio of negative ions to electrons.

The consequences of such a law are complicated, for the expression in brackets can be treated as a recombination coefficient which depends upon the electron concentration, the pressure, and possibly on the temperature, since $\lambda$ may be very sensitive to temperature. This will make the apparent value of the recombination vary with the solar activity, the season, and the time of day, but the rate of change in ionization may be roughly proportional to $N_{e}^{2}$, as is found to be the case.

The nighttime variation of $N_{e}$ for June and July (fig. 2) can be represented by the equation

$$
-\frac{d N_{e}}{d t}=0.3 \times 10^{-10} N_{e}^{2}+2 \times 10^{-5} N_{e} .
$$

This applies to a level of mean height, $360 \mathrm{~km}$. The data of figure 3 permit an evaluation of $d N_{e} / d t$ at lower levels, and indicate a value of $B p=17 \times 10^{-5}$ at $270 \mathrm{~km}$, assuming pure electron attachment. Taking the scale height as $36 \mathrm{~km}$, the pressure at $270 \mathrm{~km}$ is 12 times the pressure at $360 \mathrm{~km}$ and the attachment coefficient 8.5 times as great.

The pressure variation of electron attachment tends to make the $F$ layer rise during the night, and this is almost always observed from sunset to midnight. This pressure change complicates the problem of determining the rate of recombination and attachment. From midnight to dawn the height decreases slightly, and this is naturally ascribed to cooling of the air. However, the temperature changes introduce a further source of uncertainty in the computations. While curves of the type of figure 3 should give specific information as to the rate of recombination as a function of altitude, real or accidental variations make it impractical to evaluate the constants involved in eq 16.

\section{DAYTIME VARIATION OF ELECTRON CONCENTRATION}

The daily variation of $N_{e}$, on the basis of the simple recombination theory, has been evaluated by Millington ${ }^{10}$ for conditions comparable to October and November 1938. The broken-line curves in figure 1 show these theoretical curves, and, except for a large discrepancy in the morning, the daily variation corresponds roughly to theory. The daily curves for September, June, and July rise more abruptly at sunrise, and the maximum is near sunset. The shape of the curves suggests that the ionizing radiation is not reduced appreciably by atmospheric absorption and that the $F_{2}$ level is far above the level

10 Proc. Phys. Soc. (London) 44, 580 (1932). 
where the radiation is reduced to $1 / e$. On the assumption that the $F_{2}$ level is at a constant pressure so low that absorption is negligible and that electrons disappear by attachment,

$$
\frac{d N_{e}}{d t}=q-B p N_{e}=B p N_{\infty}-B p N_{e},
$$

where $q$ is a constant from sunrise to sunset, and $N_{\infty}$ is the equilibrium number of electrons reached after a sufficiently long time. The integral of this between times $t_{1}$ and $t_{2}$ is

$$
B p\left(t_{2}-t_{1}\right)=\ln \left(\frac{N_{\infty}-N_{1}}{N_{\infty}-N_{2}}\right)
$$

Figure 2 includes a plot of this equation for June and July, taking $N_{1}$ as the observed value at dawn, $B p=6 \times 10^{-3}$ and $N_{\infty}=0.8 \times 10^{6}$. The agreement is satisfactory, but it happens that the assumption of pure recombination gives a very similar curve. On the September curve of figure 2 the circles are computed on the assumption of pure recombination with no absorption, using the constants $\alpha^{\prime}=0.7 \times 10^{-10}$ and $N_{\infty}=1.2 \times 10^{6}$. The curves clearly indicate that the atmospheric absorption is negligible, but they do not give conclusive evidence as to the law of recombination. The surprising conclusion is, that in the summer the $F_{2}$ layer is far above the level where radiation is reduced to $1 / e$, while in the winter it is much closer to that level. From curves of Chapman ${ }^{11}$ for ion production at different heights, it is estimated that if absorption is negligible within an hour of sunrise, then the $F_{2}$ level is more than 3 times the scale height, or over $100 \mathrm{~km}$ above the level where radiation is reduced to $1 / e$. This rough estimate supports Bradbury's suggestion that $F_{1}$ and $F_{2}$ come from the same ionization process with the maximum rate of production of ions at the $F_{1}$ level.

\section{PRESSURE AT THE $F_{2}$ LEVEL}

It is convenient to determine the pressure at which $N_{e}$ is a maximum rather than the height. For midday conditions the number of electrons as a function of pressure can be expressed.

$$
\begin{gathered}
\alpha^{\prime} N_{e}^{2}=\frac{K p}{p_{o}} e^{-p / p_{0}} \\
\alpha^{\prime}=\alpha(1+\lambda)+\frac{B p}{N_{e}},
\end{gathered}
$$

where $p_{0}$ is the pressure at the level where radiation is reduced to $1 / e$. As a simple working hypothesis, assume that $\lambda$ is proportional to $p(\lambda=c p)$. The condition that there be a maximum value of $N_{e}$ at a pressure $p$ is that $N_{e}^{2} d \alpha^{\prime} / d p$ is equal to the derivative of the right side of eq $20, N_{e}$ being taken as a constant.

$$
\begin{gathered}
\left(\alpha c+\frac{B}{N_{e}}\right) N_{e}^{2}=\frac{K e^{-p / p_{0}}}{p_{0}}\left(1-\frac{p}{p_{0}}\right) \\
\alpha c p+\frac{B p}{N_{e}}=\alpha^{\prime}\left(1-\frac{p}{p_{0}}\right)
\end{gathered}
$$

11 Proc. Phys. Soc. (London) 43, 26 (1931). 


$$
\frac{p}{p_{o}}=\frac{\alpha}{\alpha^{\prime}},
$$

where $p$ is the pressure at the $F_{2}$ maximum, and $p_{0}$ is the pressure at the level where the radiation is reduced to $1 / e$. This would indicate that if the $F_{2}$ level is far above $p_{0}$, then the effective recombination coefficient, $\alpha^{\prime}$, is much greater than the electronic recombination coefficient. However, the large seasonal change of $p / p_{0}$ is not explained, for observed variations of $\alpha^{\prime}$ are in the wrong direction. In general, if $\lambda$ is not a linear function, $p / p_{0}$ will be a function of $\lambda$ and, as $\lambda$ is very sensitive to temperature, a seasonal variation can be understood. A physically simple assumption is that formation of negative ions by attachment and disappearance by recombination are in equilibrium.

$$
\begin{gathered}
\lambda(1+\lambda) \alpha_{i} N_{e}^{2}=B p N_{e} \\
\frac{d \lambda}{d p}=\frac{B}{(1+2 \lambda) \alpha_{i} N e}
\end{gathered}
$$

The substitution of this value of $d \lambda / d p$ in place of $c$ in eq 22 gives, instead of eq 23 , the relation

$$
\underline{p}=\frac{(1+\lambda)^{2} \alpha}{(1+2 \lambda) \alpha^{\prime}}
$$

If $\lambda$ is small ( 1 or less), eq 26 is nearly identical to eq 23 , but, in contrast to eq 23 , a seasonal variation in $p / p_{0}$ can here be ascribed to $\lambda$. There is no reason to believe that either equation is rigorous, but the derivations at least show, in a qualitative way, that there can be a maximum value of $N_{e}$ far above $p_{0}$. It is not true, as has been suggested, ${ }^{12}$ that electronic recombination is predominant at this maximum, but, on the contrary, it must be a small fraction of the apparent recombination coefficient, $\alpha^{\prime}$, if $p / p_{0}$ is very small. It seems probable that the vertical variations of $\lambda$ and $d \lambda / d p$ are subject to random variations and cannot be expressed as simple analytical functions. This would explain the large fluctuations in $N_{e}$ and in the height of $F_{2}$, which are commonly but not always observed. However, Appleton's assumption that $\lambda$ reaches a constant value at the $F_{1}$ level must be preserved for the reasons which he has given.

\section{NUMERICAL RESULTS}

It will be assumed, following Bradbury's suggestion, that $p_{0}$ is at the $F_{1}$ level and that the ionization comes from atomic oxygen. Then the partial pressure for June and July noon is $1.48 \times 10^{-6} \mathrm{~mm}$ at $240 \mathrm{~km}$ and (from section III) the effective temperature between 100 and 240 $\mathrm{km}$ is $680^{\circ} \mathrm{K}$; the scale height, $36 \mathrm{~km}$. This scale height will be used above $240 \mathrm{~km}$ for want of further data. In June and July the $F_{2}$ level is at $360 \mathrm{~km}, p / p_{0}=0.027$, and $p=4 \times 10^{-8} \mathrm{~mm} \mathrm{Hg}$. If it is assumed that $\lambda$ is 1 or less, eq 23 is a good approximation, and on the basis $\alpha^{\prime}=0.8 \times 10^{-10}$, it follows that $\alpha$, the coefficient of electronic recombination, is $2 \times 10^{-12}$ (eq 26 indicates that $\alpha$ may be less).

12 N. E. Bradbury, Ter. Mag. and Atmos. Elec. 43, 55 (1938). 
A recent theoretical computation ${ }^{13}$ of the recombination coefficient for atomic oxygen gives almost exactly this value; namely, $2.12 \times 10^{-12}$ at $500^{\circ} \mathrm{K}$. It would appear then that in this case $\lambda$ must bersmall, and in the expression for $\alpha^{\prime}$ electron attachment must be predominant. For October $p / p_{0}$ is larger and $\lambda$ is evidently not negligible.

The measured rate of production of ions at the $F_{2}$ level for June and July is 47 per cubic centimeter per second. It follows from the above values of $p$ and $p_{0}$ in eq 3 that the rate of production at $F_{1}\left(p=p_{0}\right)$ is 640 per $\mathrm{cm}^{3}$ per sec. This and the observed value of $N_{e}$ give $\alpha^{\prime}=5 \times 10^{-9}$ at $F_{1}$. An independent estimate of $\alpha^{\prime}$ at $F_{1}$ can be made with the approximation that electron attachment is predominant. The value $B p=6 \times 10^{-5}$ at $F_{2}\left(p=4 \times 10^{-8} \mathrm{~mm}\right)$ gives $\alpha^{\prime}=$ $B p / N_{e}=6.2 \times 10^{-9}$ at $p=1.48 \times 10^{-6} \mathrm{~mm}$, in good agreement with the first value.

The outstanding anomaly of the $F_{2}$ layer has been the seasonal variation of $N_{e}$, but this can be entirely accounted for by the seasonal variation in pressure. The maximum rate of production of ions, $\alpha^{\prime} N_{e}^{2}$, for October is 5.8 times the rate for July instead of 0.7 times the July value, as predicted by conventional theory. Since this rate is proportional to $p$ exp. $\left(-p / p_{0}\right)$, then the pressure at $F_{2}$ is 7.3 times the July value. The observed difference in height is $70 \mathrm{~km}$, and if the scale height is $36 \mathrm{~km}$, this would correspond to a pressure ratio of 7.0 .

While this pressure variation has been ascribed to a seasonal variation in $\lambda$, there is no basis for quantitative predictions. For temperature equilibrium between oxygen atoms, electrons, and ions, the ratio of ions to electrons would be given by Saha's equation with the electron affinity, 2.2 volts, in place of the ionization potential.

$$
\frac{N_{\mathrm{e}} N_{\mathrm{o}}}{N_{-}}=\frac{N_{\mathrm{o}}}{\lambda}=6.05 \times 10^{15} T^{3 / 2} e^{-2.2 \times 11600 / T},
$$

where $N_{\mathrm{o}}$ is the number of oxygen atoms per cubic centimeter. At a partial pressure of $4 \times 10^{-8} \mathrm{~mm}, \lambda$ would become unity at $960^{\circ} \mathrm{K}$, and at this temperature a 10-percent change in $T$ would give a tenfold change in $\lambda$. Oxygen ions exposed to sunlight cannot be considered to be in temperature equilibrium, and $\lambda$ is probably much less than the value given by eq 27 . The assumptions that $\lambda$ is 1 or less at a pressure of $4 \times 10^{-8} \mathrm{~mm}$, and larger but variable at 7 times this pressure, are not incompatible with eq 27 though there is no good basis for numerical computations.

\section{CONCLUSION}

To summarize the results there is little doubt that the rate of recombination decreases rapidly with decreasing pressure above $F_{1}$ and a necessary consequence of this is that the maximum number of electrons is far above the level where the rate of production is a maximum. The analysis given here involves simplifying assumptions that may well be open to question, but several independent lines of evidence indicate that the maximum rate of production must be near the $F_{1}$ level so that one absorption process can account for both

${ }^{18}$ D. R. Bates, R. A. Buckingham, H. S. W. Massey, and J. J. Unwin, Proc. Roy. Soc. (London) [A] 170, 322 (1939). 
$F$ layers. This avoids the assumption that the temperature above $F_{1}$ is extremely high and subject to enormous seasonal variations. A complication in the theory is that the height of $F_{2}$ and the electron concentration depend on the ratio of electrons to negative ions, which is extremely sensitive to temperature. Small changes in the vertical temperature gradient could produce large fluctuations in the $F_{2}$ layer, and it is possible that this can explain the apparently erratic variations in ionization that are characteristic of the $F_{2}$ layer.

One consequence of the theory is, that the variation of ionization with time and height will depend on the absolute intensity of the ionizing radiation, which varies over a wide range during a sunspot cycle. In periods of low solar activity, with low values of $N_{e}$, the electron attachment becomes relatively more important, the maximum rises to a lower pressure, and even in winter the $F_{1}$ layer appears as a separate maximum. $N_{e}$ for the $F_{1}$ layer should vary as the square root of the intensity of the ionizing radiation. In the $F_{2}$ layer the relation between $N_{e}$ and intensity is more complicated, but for summer conditions (electron attachment predominant) it will vary nearly as the first power of the intensity. ${ }^{\text {14 }}$ From July 1934 to July 1938 the $F_{1}$ ionizatoin increased by the factor 1.54 , the $F_{2}$ ionization by the factor 2.34, which is almost exactly the square of 1.54 .

The values of $B p=6 \times 10^{-5}$ at $p=4 \times 10^{-8} \mathrm{~mm}$ and $T=680^{\circ} \mathrm{K}$ lead to a value of the effective collision area, $\sigma$, for electron attachment to atomic oxygen of $6.5 \times 10^{-21} \mathrm{~cm}^{2}$. Massey ${ }^{15}$ has published a rough theoretical estimate for this constant of $2.5 \times 10^{-22} \mathrm{~cm}^{2}$ at $680^{\circ} \mathrm{K}$. This agreement is poor, but both values are subject to considerable uncertainty. As noted above, the computed value of the electronic recombination coefficient, $2 \times 10^{-12}$, is in accurate agreement with theory, though the uncertainty in this case also is large. The effective collision area for recombination at $680^{\circ} \mathrm{K}$ is $1.2 \times 10^{-19} \mathrm{~cm}^{2}$.

The author ${ }^{16}$ measured the rate of recombination in low-pressure cesium and mercury discharges and obtained values of $\alpha=2$ or $3 \times 10^{-10}$. Doubt was expressed as to whether this was the coefficient for spontaneous recombination, but the fact that $\alpha^{\prime}$ for the $F_{2}$ layer is of the same magnitude has been quoted as evidence that $\alpha^{\prime}$ was the electronic recombination coefficient. This is evidently not the case, and the ionosphere results seem to be consistent with the recombination theory.

Washington, June 8, 1940.

\footnotetext{
14 In a previous publication the author has considered that $N^{2}$. was a measure of the ionizing intensity both for $F_{1}$ and $F_{9}$, but this is inconsistent with the present theory. See Science 90, 137 (1939).

15 Proc. Roy. Soc. (London) [A] 163, 542 (1937).

16 J. Research NBS 19,447 (1937) RP1037; 19,559 (1937), RP1045. There is a numerical error in the theoretical value of $\alpha$ used in the second paper.
} 